\title{
COMMISSIONING OF THE NEW MULTIPOLE WIGGLERS IN THE SRS
}

\author{
J. A. Clarke and G. S. Dobbing* \\ CLRC Daresbury Laboratory, Daresbury, Warrington. WA4 4AD, UK
}

\begin{abstract}
Two new high field $2 \mathrm{~T}$ permanent magnet multipole wigglers have been installed into the SRS. This paper describes the effect of the new insertion devices on the SRS lattice. Closed orbit distortion, betatron tune change and emittance blow up have all been measured and the results are compared with lattice model predictions which are based on actual measured magnetic field values.
\end{abstract}

\section{INTRODUCTION}

The 1998 SRS Upgrade has been completed and two new multipole wigglers (MPWs) have been installed as planned [1]. These new additions complement the two superconducting wigglers and undulator that have been in the SRS for some time. One of the wigglers will provide light for two experimental stations, both dedicated to protein crystallography [2]. The other will have one station for studying the interaction of photons with molecules, nanoclusters and surfaces although provision has been made for adding a second station at a later date. The new multipole wigglers are identical permanent magnet insertion devices optimised for output around 10 $\mathrm{keV}$. A design comprising nine $2 \mathrm{~T}$ poles and two $1.7 \mathrm{~T}$ end poles was chosen for the wigglers, yielding a 25 fold increase in photon flux per horizontal angle at $10 \mathrm{keV}$ compared to an SRS dipole.

As the SRS is a second generation light source, installing the new insertion devices necessitated the relocation of several machine components, notably all four RF cavities were moved to alternative straight sections [3]. This upgrade also provided one further free straight, possibly to be occupied in the future by a helical undulator to give light with variable polarisation. Installation of the wigglers and manoeuvring of machine components was completed in a shutdown at the end of 1998. One of the narrow gap vessels for use with the new wigglers had already been installed in January 1998 so experience could be gained with a much-reduced vertical aperture.

\section{MODELLING THE MULTIPOLE WIGGLERS}

Before the multipole wigglers were installed much commissioning work had already been carried out. Numerous protective devices had been proved to work (e.g. magnet array tilt, temperature and beam position

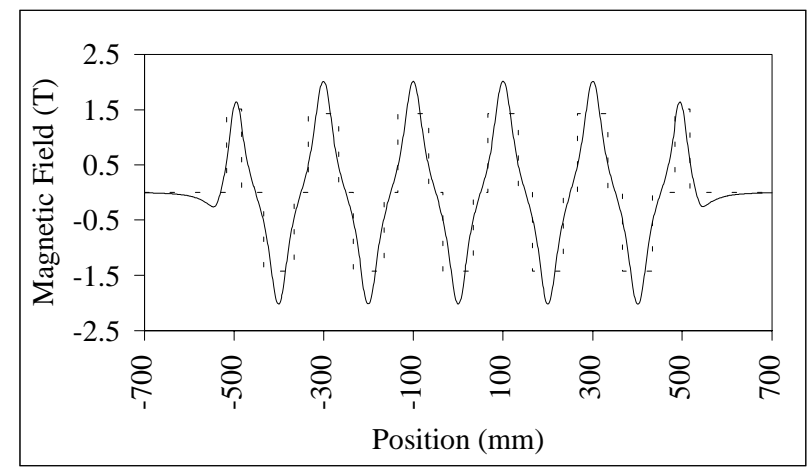

Figure 1: Measured magnetic field along the magnetic axis of an MPW at operational gap (solid line). The field assumed in the hard-edged lattice model is also shown (broken line).

interlocks) and the magnetic fields produced by the wigglers had been measured extensively [4]. These measurements were used to build a lattice model of the MPWs.

To predict the effect of the MPWs on the SRS electron beam a hard-edged model was used. The number of poles and the total length of a real MPW were conserved. Two types of pole were used; the MPW model was composed of two end poles and nine central poles, with zero-field drift spaces in between. Actual measurements of the magnetic field through an MPW are plotted in fig. 1 [4]. The magnetic field for the hard edged model is also plotted. Pole length and magnetic field in the model were varied to match integrals of powers of the on axis magnetic field. Measurements were taken from one of the MPWs, the other was found to be extremely similar. Net angle of bend produced by a magnetic field depends on the integral of the magnetic field along the electron path. Integrating the second power of the field gives the predominant focussing effect whilst change in emittance is affected primarily by the integral of the modulus of the third power of magnetic field on the electron path. The hard-edged model cannot match integrals of all three powers of magnetic field simultaneously so a model that gave the correct integrals of first and second powers was used to predict betatron tune shift. Similarly a model that matched measured integrals of the first and third power of field was used to predict emittance blow-up. It is interesting to note that this technique leads to a model in which the end poles have a higher field strength than the centre poles. This is the same approach that was used to predict the tune shift and emittance increase, accurate to

*E-mail g.s.dobbing@dl.ac.uk 
first order, due to the SRS superconducting wigglers that were installed in 1982 and 1993 [5,6].

The emittance increase and tune shift due to one of the MPWs predicted using the Daresbury lattice code "ORBIT" are shown in table 1. Values without the MPW are shown for comparison.

Table 1: Predicted emittance and tune shifts in the SRS for multibunch operations. It is estimated that an MPW at operational gap will cause an emittance increase of $4 \%$.

\begin{tabular}{|c|c|c|}
\hline & $\begin{array}{c}\text { Without } \\
\text { MPW }\end{array}$ & $\begin{array}{c}\text { MPW at operational } \\
\text { gap }\end{array}$ \\
\hline $\begin{array}{c}\text { Radial Emittance } \\
\text { (nm rad) }\end{array}$ & 104 & 108 \\
\hline Vertical Tune & 3.350 & 3.366 \\
\hline Vertical Tune Shift & 0 & 0.016 \\
\hline Horizontal Tune & 6.198 & 6.198 \\
\hline
\end{tabular}

\section{MEASURED STORAGE RING BEHAVIOUR}

\subsection{Orbit Shifts}

The first MPW was commissioned with beam in February 1999. As the magnet gap was closed the betatron tunes and closed orbit were recorded. Plots of the uncorrected RMS horizontal and vertical orbit as a function of magnet gap are shown in fig. 2. Fig. 3 shows the change in the integrated magnetic field through the MPW, measured before the MPW was installed and resulting change in the orbit at a typical horizontal beam position monitor as the MPW gap is closed. Excellent correlation is evident between the integrated field through the MPW and the resulting orbit distortion.

\subsection{Tune Shift and Beam Size.}

The SRS tune measuring system measures the coherent response of the beam to an electrostatic deflection. This signal is seen at a frequency corresponding to the fractional part of the betatron tune. Horizontal and vertical tunes were measured as the MPW magnet gap was closed. Horizontal tune was unchanged to within 0.002 ; a plot of vertical tune shift against magnet gap is shown in fig. 4. A strong correlation between the MPW peak field and the vertical tune shift is evident, as expected.

The discrepancy between the tune shift measured at the MPW minimum operating magnet gap of $20.5 \mathrm{~mm}$ and that calculated using the hard edged model is $16 \%$. When used for calculations preceding the installation of the superconducting wigglers in 1982 and 1993 the discrepancies were zero (to within the accuracy of the measurement) and $6 \%$ too high respectively $[7,8]$. These results, taken with those presented in this paper suggest that the hard edged model becomes less accurate for high or complicated fields, as may be expected for a simple model. However, the discrepancies are not so large as to

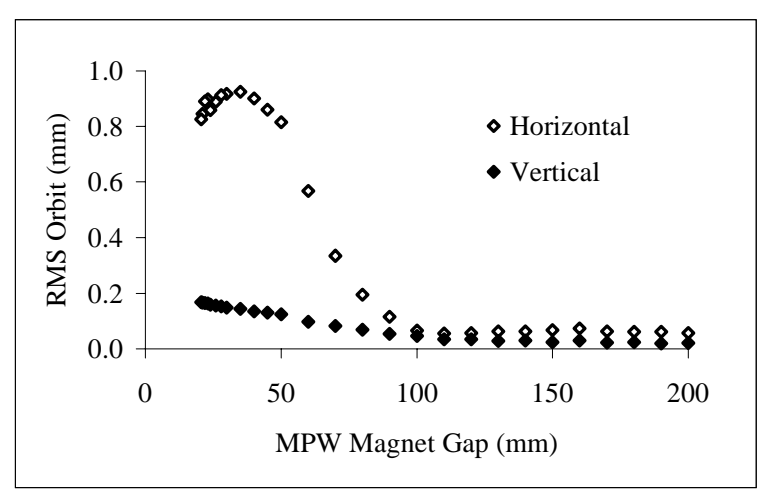

Figure 2: Horizontal and vertical RMS orbits at different MPW magnet gaps.

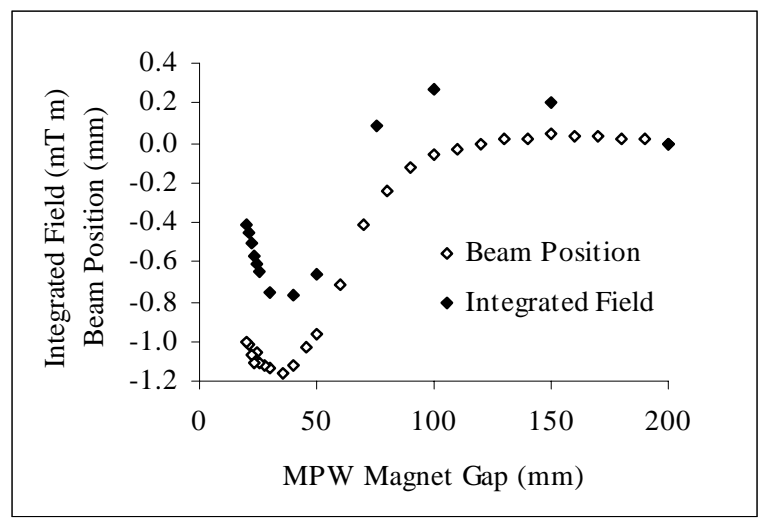

Figure 3: Changes to horizontal beam position at a typical BPM and integrated magnetic field as the MPW magnet gap is closed from $200 \mathrm{~mm}$.

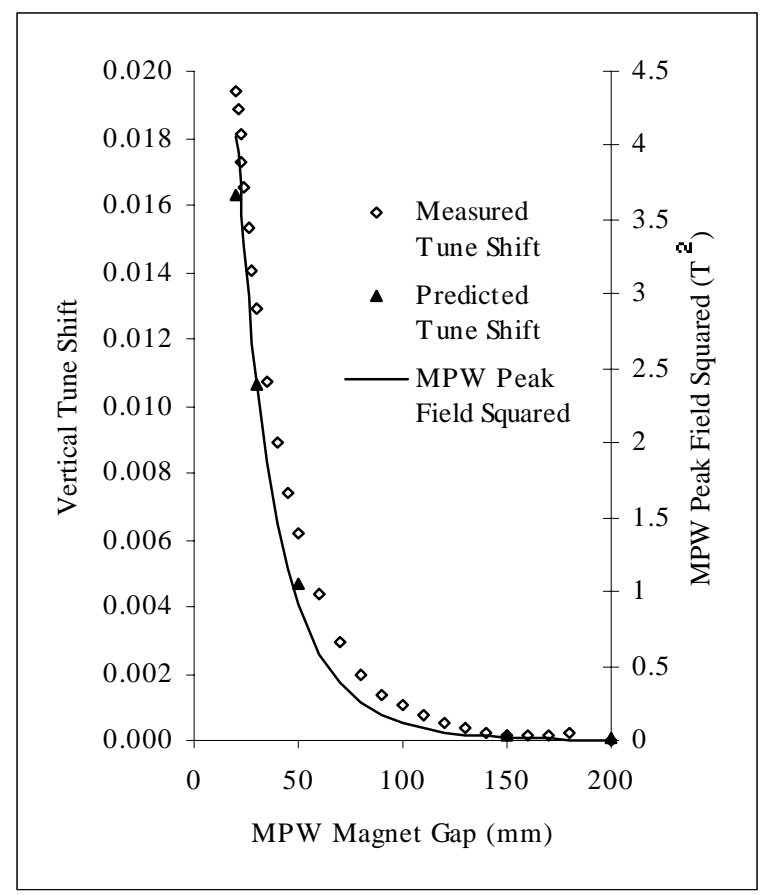

Figure 4: Measured and predicted vertical tuneshifts at different MPW magnet gaps. The MPW peak field is also shown. 
make the approach used here invalid. In general calculations are only required to give an approximate estimate of effects on the electron beam, in order to specify the accelerator components required for correction. Therefore the hard edged model is still useful to accelerator physicists as a comparatively quick and accurate way of anticipating the effects that a proposed insertion device will have on an accelerators characteristics.

During commissioning the beam sizes at the SR diagnostic port were not seen to change (to within the 5\% repeatability of the measurement). This is consistent with the very small predicted increase in the emittance, shown in table 1 . No change in horizontal-vertical coupling was observed.

The MPW was not observed to have any effect on the electron beam lifetime.

\subsection{Correcting the Effects of the MPWs on the Storage Ring}

A similar correction scheme is used for the new MPWs as was successfully implemented for the superconducting wigglers at Daresbury [6]. Trim coils fitted around the magnet arrays correct the integrated field of the MPW to minimise the RMS horizontal orbit. An active shunt is fitted to the local vertically focussing quadrupole to correct for the vertical focussing of the MPW. During the commissioning of an MPW the currents required through the trim and shunt to correct both the orbit and tunes for several MPW magnet gaps were recorded. These values were then fed into the control system so that software may be used to automatically carry out orbit and tune correction as the magnet gap is changed during operations. Initial trials of these systems were very successful.

\section{SUMMARY}

As well as preparing one MPW for use, its radiation port has also been commissioned, although the beamline and experimental stations are not due for completion until May of this year. Radiation was successfully extracted from the port on $8^{\text {th }}$ March 1999, at the first attempt, making it likely that the two new protein crystallography stations will be ready for users by September and November respectively [9]. The second MPW beamline will not be permitted to take beam until it is complete, for radiation safety reasons. This will happen during the second half of 1999.

\section{REFERENCES}

[1] Clarke, J. A., M. W. Poole, "Upgrading The Daresbury SRS With Additional Insertion Devices and its Implications For the Storage Ring Layout", Proc. $5^{\text {th }}$ Euro. Part. Accel. Conf., Sitges, p 623, 1996.

[2] Duke, E. M. H., R.C. Kehoe, P.J. Rizkallah, J.A. Clarke, C. Nave, J. Synchrotron Rad., 5, pp 497-499, 1998.

[3] Dykes, D.M., "RF System Changes Associated With the SRS Upgrade", Proc. $5^{\text {th }}$ Euro. Accel. Conf., Sitges, p1946, 1996.

[4] Clarke, J.A., N. Bliss, D.J. Bradshaw, N.W. Harris, C.L. Hodgkinson, R. Marl, I.D. Mullacrane, M.W. Poole, M.J. Pugh, R.J. Reid, "Final Measurements of the SRS Multipole Wigglers", these proceedings

[5] Marks, N., G.N. Greaves, M.W. Poole, V.P. Suller, R.P. Walker, "Initial Operation of a 5T Superconducting Wiggler Magnet in the SRS”, Nucl. Instr. And Meth. Phys. Res. 208 (1983), p 97.

[6] Poole, M.W, J.A. Clarke, P.D. Quinn, S.L. Smith, V.P. Suller, L.A. Welbourne, "Commissioning a Second Superconducting Wiggler in the Daresbury SRS", Proc. 1993 IEEE Part. Accel. Conf. Washington, 1993.

[7] Suller, V.P., N. Marks, M.W. Poole, R.P.Walker, "SRS Behaviour With a Superconducting 5T Wiggler Insertion", Proc. 1983 IEEE Part. Accel. Conf., Santa Fe, 1983.

[8] Clarke, J.A., D.M. Dykes, S.F. Hill, E.A. Hughes, P.A. McIntosh, M.W. Poole, P.D. Quinn, S.L. Smith, V.P. Suller, L.A. Welbourne, "Update on Commissioning and Operations With the Second Superconducting Wiggler at Daresbury, Proc $4^{\text {th }}$ Euro. Part. Accel. Conf., London, p648, 1994.

[9] http://www.dl.ac.uk/SRS/PX/events.html 